\title{
ON NORMAL DERIVATIONS
}

\author{
JOEL ANDERSON
}

Abstract. Let $\Delta_{T}$ be the derivation on $\mathfrak{B}(\mathscr{H})$ defined by $\Delta_{T}(X)=T X-X T(T, X \in \mathfrak{B}(\mathscr{H}))$. We prove that if $T$ is an isometry or a normal operator, then the range of $\Delta_{T}$ is orthogonal to the null space of $\Delta_{T}$. Also, we prove that if $T$ is normal with an infinite number of points in its spectrum then the closed linear span of the range and the null space of $\Delta_{T}$ is not all of $\mathfrak{B}(\mathscr{H})$.

Introduction. If $\mathscr{H}$ is a Hilbert space and $\mathfrak{B}(\mathscr{H})$ is the algebra of all bounded linear operators on $\mathscr{H}$, then for each fixed $T \in \mathfrak{B}(\mathscr{H})$ the operator equation

$$
\Delta_{T}(X)=T X-X T
$$

defines a bounded linear operator on $\mathfrak{B}(\mathscr{H}) . \Delta_{T}$ is called a derivation because, for all $X, Y$ in $\mathfrak{B}(\mathscr{H})$,

$$
\Delta_{T}(X Y)=\Delta_{T}(X) Y+X \Delta_{T}(Y) .
$$

When $N$ is a normal operator in $\mathfrak{B}(\mathscr{H})$ we will say that $\Delta_{N}$ is a normal derivation.

If $T \in \mathfrak{B}(\mathscr{H})$ has a particular property it is often the case that $\Delta_{T}$ has a similar property. For example if $T$ is selfadjoint then it is easy to show that the numerical range of $\Delta_{T}$ is real; i.e., that $\Delta_{T}$ is Hermitian in the sense of Lumer and Vidav (see [4]). Also, if $N$ is normal then it is shown in [1] that $\Delta_{N}$ is a generalized scalar operator. When $N$ is a normal operator in $\mathfrak{B}(\mathscr{H})$ with null space $\mathscr{N}(N)$ and range $\mathfrak{R}(N)$ it is elementary that

(i) $\mathfrak{R}(N) \perp \mathscr{N}(N)$,

(ii) $\mathfrak{R}(N)^{-} \oplus \mathscr{N}(N)=\mathscr{H}$.

In this note we study the extent to which $\Delta_{N}$ shares these properties. We find that the range $\Re\left(\Delta_{N}\right)$ and the null space $\mathscr{N}\left(\Delta_{N}\right)$ are "orthogonal" in a certain sense so that (i) holds, but that (ii) holds if and only if the spectrum of $N$ contains only a finite number of points. In the last section we mention some open questions.

Received by the editors September 21, 1970 and, in revised form, July 14, 1972. AMS (MOS) subject classifications (1969). Primary 4710, 4755; Secondary 4610.

Key words and phrases. Derivation, commutant of a normal operator, commutant of an isometry, orthogonality, complemented subspace.

(c) American Mathematical Society 1973 
(1.1) Notation. $\Re\left(\Delta_{T}\right)$ is the (not necessarily closed) set of operators in $\mathfrak{B}(\mathscr{H})$ of the form $\Delta_{T}(X)$ when $X \in \mathfrak{B}(\mathscr{H})$. Note that the null space $\mathscr{N}\left(\Delta_{T}\right)$ is just the commutant of $T$.

(1.2) Definition. Let $\boldsymbol{C}$ be the complex numbers and let $\mathfrak{X}$ be a normed linear space. Let $x, y \in \mathfrak{X}$. If $\|x-\lambda y\| \geqq\|\lambda y\|$ for all $\lambda \in C$ then $x$ is said to be orthogonal to $y$. Let $\mathscr{M}$ and $\mathscr{N}$ be two subspaces in $\mathfrak{X}$. If $\|m+n\| \geqq\|n\|$ for all $m \in \mathscr{M}$ and for all $n \in \mathscr{N}$ then $\mathscr{M}$ is said to be orthogonal to $\mathscr{N}$.

(1.3) REMARK. This definition generalizes the idea of orthogonality in Hilbert space. (It is not new. See [5] for example.) Note that in general $x$ orthogonal to $y$ does not imply $y$ orthogonal to $x$. Also it is easy to show that if $\mathscr{M}$ and $\mathscr{N}$ are closed subspaces of $\mathfrak{X}$ and $\mathscr{M}$ is orthogonal to $\mathscr{N}$ then the algebraic direct sum $\mathscr{M} \dot{+} \mathscr{N}$ is a closed subspace of $\mathfrak{X}$.

(1.4) THEOREM. Let $S$ be an isometry in $\mathfrak{B}(\mathscr{H})$. Then $\Re\left(\Delta_{S}\right)$ is orthogonal to $\mathscr{N}\left(\Delta_{S}\right)$.

Proof. From [3, Problem 185] we know

$$
\sum_{i=0}^{n-1} S^{n-i-1}(S X-X S) S^{i}=S^{n} X-X S^{n}
$$

Thus if $S T=T S$,

so

$$
n S^{n-1} T=S^{n} X-X S^{n}-\sum_{i=0}^{n=1} S^{n-i-1}(S X-X S-T) S^{i},
$$

$$
\|T\|=\left\|S^{n} T\right\| \leqq(1 / n)\left\|S^{n} X-X S^{n}\right\|+\|S X-X S-T\| .
$$

The result now follows by letting $n \rightarrow \infty$.

(1.5) TheORem. Let $A$ be a selfadjoint operator in $\mathfrak{B}(\mathscr{H})$. Then $\Re\left(\Delta_{A}\right)$ is orthogonal to $\mathscr{N}\left(\Delta_{A}\right)$.

Proof. Let $U=(A-i)(A+i)^{-1}$ be the Cayley transform of $A$. Then $U$ is unitary and $A=i(1+U)(1-U)^{-1}$. Now if $X \in \mathfrak{B}(\mathscr{H})$,

Hence

$$
\begin{aligned}
\Delta_{A}(X) & =(A-i) X-X(A-i)=U(A+i) X-X(A+i) U \\
& =\Delta_{U}((A+i) X)+\Delta_{A+i}(X U) .
\end{aligned}
$$

$$
\Delta_{A}(X(1-U))=\Delta_{U}((A+i) X) .
$$

Since $1-U$ and $A+i$ are both invertible, $\Re\left(\Delta_{A}\right)=\Re\left(\Delta_{U}\right)$. Also it is clear that $A T=T A$ implies $U T=T U$ so that (1.4) applies and the result follows.

(1.6) Lemma. Let $P_{1}, \cdots, P_{n}$ be orthogonal idempotents (i.e. $P_{i} P_{j}=0$ if $i \neq j$ and $P_{i}^{2}=P_{i}$ for $\left.i=1, \cdots, n\right)$. Let $\left\{\lambda_{1}, \cdots, \lambda_{n}\right\}$ and $\left\{\mu_{1}, \cdots, \mu_{n}\right\}$ be sets 
of nonzero complex numbers such that $\lambda_{i} \neq \lambda_{j}$ and $\mu_{i} \neq \mu_{j}$ if $i \neq j$. Let

Then $\mathfrak{R}\left(\Delta_{Q_{1}}\right)=\mathfrak{R}\left(\Delta_{Q_{2}}\right)$.

$$
Q_{1}=\sum_{i=1}^{n} \lambda_{i} P_{i}, \quad Q_{2}=\sum_{i=1}^{n} \mu_{i} P_{i} .
$$

Proof. Let $P_{0}=1-\sum_{i=1}^{n} P_{i}, \lambda_{0}=\mu_{0}=0$, let $X \in \mathfrak{B}(\mathscr{H})$. Then a simple computation shows

$$
\begin{aligned}
& \Delta_{Q_{1}}(X)=\sum_{i=0}^{n} \sum_{j=0}^{n}\left(\lambda_{i}-\hat{\lambda}_{j}\right) P_{i} X P_{j}, \\
& \Delta_{Q_{2}}(X)=\sum_{i=0}^{n} \sum_{j=0}^{n}\left(\mu_{i}-\mu_{j}\right) P_{i} X P_{j},
\end{aligned}
$$

and since $\lambda_{i} \neq \lambda_{j}$ and $\mu_{i} \neq \mu_{j}$ if $i \neq j$ the assertion is now clear.

(1.7) THEOREM. Let $N$ be a normal in $\mathfrak{B}(\mathscr{H})$ with spectral measure $E(\cdot)$. Then for all $X \in \mathfrak{B}(\mathscr{H})$ and for all $T \in \mathscr{N}\left(\Delta_{N}\right)$,

$$
\left\|T-\Delta_{N}(X)\right\| \geqq\|T\| \text {. }
$$

That is, $\mathfrak{R}\left(\Delta_{N}\right)$ is orthogonal to $\mathscr{N}\left(\Delta_{N}\right)$.

Proof. By the spectral theorem it is sufficient to show that

$$
\left\|T-\left(\sum_{i=1}^{n} \lambda_{i} E\left(\delta_{i}\right)\right) X-X\left(\sum_{i=1}^{n} \lambda_{i} E\left(\delta_{i}\right)\right)\right\| \geqq\|T\|
$$

holds for all $X \in \mathfrak{B}(\mathscr{H})$, for all $T \in \mathscr{N}\left(\Delta_{N}\right)$, for every disjoint collection $\left\{\delta_{i}\right\}_{i=1}^{n}$ of Borel sets and for every collection $\left\{\lambda_{i}\right\}_{i=1}^{n}$ of complex numbers. Further, we may assume that $\lambda_{i} \neq \lambda_{j}$ if $i \neq j$. Now let

$$
Q_{1}=\sum_{i=1}^{n} \lambda_{i} E\left(\delta_{i}\right), \quad Q_{2}=\sum_{i=1}^{n} i E\left(\delta_{i}\right) .
$$

Then $\Re\left(\Delta_{Q_{1}}\right)=\Re\left(\Delta_{Q_{2}}\right)$ by (1.6). But $Q_{2}$ is selfadjoint and $T \in \mathscr{N}\left(\Delta_{N}\right)$ implies that $T \in \mathscr{N}\left(\Delta_{Q_{i}}\right), i=1,2$. (Recall that if $T$ commutes with a normal operator $N$ it commutes with each of the spectral projections associated with $N$. This fact will be used in the proof of (2.2) below.)

(2.1) REMARK. In view of the foregoing, one might be led to believe that when $N$ is normal

$$
\mathfrak{R}\left(\Delta_{N}\right)^{-}+\mathscr{N}\left(\Delta_{N}\right)=\mathfrak{B}(\mathscr{H})
$$

where $\mathfrak{R}\left(\Delta_{N}\right)^{-}$is the uniform closure. It seems somewhat surprising that when $\mathscr{H}$ is infinite dimensional this occurs only in very special cases. 
(2.2) THEOREM. Let $\mathscr{H}$ be an infinite dimensional Hilbert space. Let $N$ be a normal operator in $\mathfrak{B}(\mathscr{H})$ with spectral measure $E(\cdot)$. If the spectrum $\sigma(N)$ of $N$ contains an infinite number of points, then there is an operator $V \in \mathfrak{B}(\mathscr{H})$ such that $\mathfrak{R}\left(\Delta_{N}\right)^{-}+\mathscr{N}\left(\Delta_{N}\right)$ is orthogonal to $V$. If $\mathscr{H}$ is separable or if $N$ has an infinite number of eigenvalues $V$ may be taken to be an isometry.

Proof. Suppose first $N$ has a finite number of eigenvalues. Let $P_{0}$ be the projection onto the span of the eigenvectors of $N$ and consider $N^{\prime}=$ $\left(1-P_{0}\right) N\left(1-P_{0}\right)$. Then $\sigma\left(N^{\prime}\right)$ is infinite so we may choose a Cauchy sequence of distinct points $\lambda_{n} \in \sigma\left(N^{\prime}\right)$. Let $r_{n}=\inf _{m \neq n}\left|\lambda_{m}-\lambda_{n}\right|$. By passing to a subsequence if necessary we may assume that $\lambda_{n} \notin \sigma\left(P_{0} N P_{0}\right)$ and $r_{n}>0$ for $n=1,2, \cdots$. Note that $r_{n} \rightarrow 0$ as $n \rightarrow \infty$. Let $\delta_{n}$ be the open disc of radius $r_{n} / 3$ about $\lambda_{n}$. The $\delta_{n}$ are disjoint and $E_{n}=E\left(\delta_{n}\right)$ are orthogonal. Note that $E_{n} \mathscr{H}$ is nonzero because $\lambda_{n} \in \delta_{n}$ and the dimension of $E_{n} \mathscr{H}$ is infinite because $N^{\prime}$ has no eigenvalues. Now let $U_{n}$ be a norm 1 transformation from $E_{n} \mathscr{H}$ into $E_{n+1} \mathscr{H}$. Note that if $\mathscr{H}$ is separable the dimension of $E_{n} \mathscr{H}^{\prime}$ is the same as the dimension of $E_{n+1} \mathscr{H}$ and $U_{n}$ may be taken to be unitary. Now define $V$ as follows: Let $\mathscr{H}=\mathscr{H}_{0} \oplus \mathscr{H}_{1} \oplus \cdots$ where $\mathscr{H}_{n}=E_{n} \mathscr{H}$ for $n=1,2, \cdots$ and $\mathscr{H}_{0}$ is the orthogonal complement of $\mathscr{H}_{1} \oplus \mathscr{H}_{2} \oplus \cdots$. Let $V$ be identity on $\mathscr{H}_{0}$ and for $x \in \mathscr{H}_{n}$ let $V x=U_{n} x$. Clearly if $\mathscr{H}$ is separable $V$ may be taken to be an isometry. Now from the choice of $E_{n}$ and the spectral theorem we know

$$
\left\|N E_{n}-\lambda_{n} E_{n}\right\|=\left\|E_{n} N-\lambda_{n} E_{n}\right\|<r_{n} / 3 .
$$

Now let $X \in \mathfrak{B}(\mathscr{H}), T \in \mathscr{V}\left(\Delta_{N}\right)$ and let $\alpha=\left\|V-\Delta_{N}(X)-T\right\|$. Thus

$$
\begin{gathered}
\alpha=\left\|E_{n+1}\right\|\left\|V-\Delta_{N}(X)-T\right\|\left\|E_{n}\right\|, \\
\alpha \geqq\left\|E_{n+1} V E_{n}-E_{n+1}\left(\Delta_{. V}(X)\right) E_{n}\right\| \quad\left(\text { since } E_{n+1} T E_{n}=E_{n+1} E_{n} T=0\right)
\end{gathered}
$$

and

so

$$
1-\alpha \leqq\left\|E_{n+1} N X E_{n}-E_{n+1} X N E_{n}\right\| \quad\left(\text { since }\left\|E_{n+1} V E_{n}\right\|=1\right)
$$

$1-\alpha \leqq\left\|N E_{n+1} X E_{n}-\lambda_{n+1} E_{n+1} X E_{n}\right\|+\left\|\lambda_{n} E_{n+1} X E_{n}-E_{n+1} X E_{n} N\right\|$

$$
+\left\|\left(\lambda_{n+1}-\lambda_{n}\right) E_{n+1} X E_{n}\right\| \text {. }
$$

Therefore

$$
1-\alpha \leqq\left(r_{n} / 3+r_{n+1} / 3+\left|\lambda_{n+1}-\lambda_{n}\right|\right)\|X\| \text {. }
$$

Letting $n \rightarrow \infty$ the right-hand side of (1) goes to 0 . Hence $\alpha \geqq 1$.

Now suppose $N$ has an infinite number of eigenvalues. Choose $\left\{\lambda_{n}\right\}_{n=1}^{\infty}$ a Cauchy sequence of distinct eigenvalues of $N$. Let $\left\{x_{n}\right\}_{n=1}^{\infty}$ be such that 
$N x_{n}=\lambda_{n} x_{n}$. Let $\mathscr{H}_{n}=$ span of $x_{n}$ for $n=1,2, \cdots$ and let $\mathscr{H}_{0}$ be the orthogonal complement of $\mathscr{H}_{1} \oplus \mathscr{H}_{2} \oplus \cdots$. Let $V$ be the identity on $\mathscr{H}_{0}$ and let $V x_{n}=x_{n+1}$ for $n \geqq 1$. Clearly $V$ is an isometry. From this point on the proof is the same as before.

(2.3) REMARKS. If $P=\left(\begin{array}{ll}1 & 0 \\ 0 & 0\end{array}\right)$ is the projection onto $\mathscr{H}_{1}$ with null space $\mathscr{H}{ }_{2}, \mathscr{H}=\mathscr{H}_{1} \oplus \mathscr{H}_{2}$, then

$$
\left(\begin{array}{ll}
1 & 0 \\
0 & 0
\end{array}\right)\left(\begin{array}{cc}
W & X \\
Y & Z
\end{array}\right)-\left(\begin{array}{cc}
W & X \\
Y & Z
\end{array}\right)\left(\begin{array}{ll}
1 & 0 \\
0 & 0
\end{array}\right)=\left(\begin{array}{cc}
0 & X \\
-Y & 0
\end{array}\right)
$$

and it is clear that

Thus in this case

$$
\mathscr{N}\left(\Delta_{P}\right)=\left\{\left(\begin{array}{cc}
W & 0 \\
0 & Z
\end{array}\right): W \in \mathfrak{B}\left(\mathscr{H}_{1}\right), Z \in \mathfrak{B}\left(\mathscr{H}_{2}\right)\right\} .
$$

$$
\mathfrak{R}\left(\Delta_{N}\right)+\mathscr{N}\left(\Delta_{N}\right)=\mathfrak{B}(\mathscr{H}) .
$$

Slightly more complicated computations show that if $Q=\sum_{i=1}^{n} \lambda_{i} P_{i}$ where $P_{i}$ are mutually orthogonal selfadjoint projections then again

$$
\mathfrak{R}\left(\Delta_{Q}\right)+\mathscr{N}\left(\Delta_{Q}\right)=\mathfrak{B}(\mathscr{H}) \text {. }
$$

Since a normal operator has a finite number of points in its spectrum if and only if it is a finite linear combination of orthogonal selfajdoint projections, we have proved the converse to (2.2) which we record below.

(2.4) TheOREM. Let $N$ be a normal operator in $\mathfrak{B}(\mathscr{H})$. Then $\mathfrak{R}\left(\Delta_{N}\right)^{-} \dot{+}$ $\mathscr{V}\left(\Delta_{.}\right)=\mathfrak{B}(\mathscr{H})$ if and only if the spectrum of $N$ consists of a finite number of points.

(3.1) Comments and Questions. The term "normal derivation" may be justified as follows. We may define a "quasi-adjoint" to $\Delta_{T}$ by

$$
\Delta_{r}^{*}(X)=\left(\Delta_{T}\left(X^{*}\right)\right)^{*}=\Delta_{-T^{*}}(X) \text {. }
$$

Then since $\Delta_{A} \Delta_{B}-\Delta_{B} \Delta_{A}=\Delta_{A B-B A}$ and 1 is not a commutator $\Delta_{T}^{*} \Delta_{T}=$ $\Delta_{T} \Delta_{T}^{*}$ if and only if $T^{*} T=T T^{*}$.

We now know that the range of a derivation induced by an isometry or a normal operator is orthogonal to its null space. Simple $2 \times 2$ matrix examples show that this is not the case for nilpotent operators. (In fact, if $T^{2}=0$ there is an $X \in \mathfrak{B}(\mathscr{H})$ such that $\Delta_{T}(X)=T$.)

It is known (see [2]) that if $N$ is normal then $\mathscr{N}\left(\Delta_{N}\right)$ is complemented in $\mathfrak{B}(\mathscr{H})$. On the other hand by $(2.4)$ it is in general false that $\mathfrak{R}\left(\Delta_{\mathrm{N}}\right)^{-} \dot{+}$ $\mathscr{N}^{\prime}\left(\Delta_{N}\right)=\mathfrak{B}(\mathscr{H})$. Hence, the following questions arise;

(i) Is there a simple property which characterizes those operators in the span of $\mathfrak{R}\left(\Delta_{N}\right)$ and $\mathscr{N}\left(\Delta_{N}\right)$ ?

(ii) What is an orthogonal complement of $\mathscr{N}\left(\Delta_{N}\right)$ ? 


\section{REFERENCES}

1. 1. Colojoară and C. Foiaş, Theory of generalized spectral operators, Gordon and Breach, New York, 1968.

2. J. Hakeda and J. Tomiyama, On some extension properties of von Neumann algebras, Tôhoku Math. J. (2) 19 (1967), 315-323. MR 36 \#5706.

3. P. R. Halmos, A Hilbert space problem book, Van Nostrand, Princeton, N.J., 1967. MR 34 \#8178.

4. G. Lumer, Semi-inner-product spaces, Trans. Amer. Math. Soc. 100 (1961), 29-43. MR 24 \#A2860.

5. J. P. Williams, Finite operators, Proc. Amer. Math. Soc. 26 (1970), 129-136. MR 41 \#9039.

Department of Mathematics, Indiana University, Bloomington, indiana 47401

Current address: Department of Mathematics, California Institute of Technology, Pasadena, California 91109 